\title{
CRACKS OF HIGHER MODES IN COSSERAT CONTINUA
}

\author{
E. Pasternak ${ }^{1}$, A.V. Dyskin ${ }^{1}$ and H.-B. Mühlhaus ${ }^{2}$ \\ ${ }^{1}$ Department of Civil and Resource Engineering, University of Western Australia, Australia \\ ${ }^{2}$ Department of Earth sciences, University of Queensland, Australia \& CSIRO Division of Exploration and Mining, \\ Australian Resource Research Centre, Australia
}

\section{ABSTRACT}

The paper considers a special type of failure in layered materials with sliding layers that develops as a progressive breakage of layers forming a narrow zone. This zone propagates as a "bending crack", ie a crack that can be represented as a distribution of disclinations. This situation is analysed using a 2D Cosserat continuum model. Edge dislocations (displacement discontinuities) and a disclination (the discontinuity in the derivative of layer deflection) are considered. The disclination does not create shear stresses along the axis perpendicular to the direction of layering, while the dislocation does not create a moment stress along the same axis. Semi-infinite and finite bending cracks normal to layering are considered. The moment stress concentration at the crack tip has a singularity of the power $-1 / 4$.

\section{INTRODUCTION}

This paper considers fracture of layered materials consisting of many layers that are thin compared to the characteristic size of the loading (eg, wave length). In this case the explicit modelling of fracturing of every layer becomes cumbersome. An alternative technique would be in representing the material as an equivalent continuum that provides a large-scale (average) description of the material response to loading. This is achieved by introducing volume elements that are much greater than the layer thickness and, at the same time, much smaller than the characteristic length of stress variations. It should be noted that in the framework of this approach no lengths smaller than the volume element size can be distinguished.

Modelling materials with microstructure requires in some cases the introduction of continua with additional degrees of freedom, such as Cosserat continuum. An example is a layered material in the case when slip between the layers is permitted. Then independent bending of layers introduces another degree of freedom associated with the field of rotations of central axes of the layers independent of macroscopic displacement field. Such a material can macroscopically be modelled by an anisotropic Cosserat continuum, a 2D version of which was considered in [1-3]. In this case the independent Cosserat rotation is represented by gradient of deflection, while the moment stress corresponds to the bending moment per unit area in the layer cross-section. Under this approximation the dislocations, disclination and fractures will be investigated representing different types of material failure. The Cosserat continuum is characterized by the presence of three additional degrees of freedom corresponding to three components of independent Cosserat rotation. Consequently, more crack modes can exist. These cracks can be envisaged as discontinuities in the corresponding components of the Cosserat rotation and modelled as distributions of disclinations (or disclination loops). The criteria of growth can then be formulated by generalizing the J-integral concept. Pasternak [4] considered dislocations, disclinations and semi-infinite cracks in layered materials in the $2 \mathrm{D}$ Cosserat approximation. We provide highlights of the Fracture Mechanics of layered materials here. 


\section{MODEL OF LAYERED MATERIAL BASED ON COSSERAT CONTINUUM}

Let us consider a 2D approximation referred to a cross section normal to layers. Let the $x$-axis of the Cartesian co-ordinate set $(x, y)$ be directed parallel to the layers. Then the behaviour of the corresponding Cosserat continuum will be governed by the following equations $[1,2,4]$ :

the equilibrium equations with body force $\left(f_{x}, f_{y}\right)$ and body moment $m_{z}$

$$
\frac{\partial \sigma_{x x}}{\partial x}+\frac{\partial \sigma_{y x}}{\partial y}+f_{x}=0, \frac{\partial \sigma_{x y}}{\partial x}+\frac{\partial \sigma_{y y}}{\partial y}+f_{y}=0, \frac{\partial \mu_{x z}}{\partial x}+\sigma_{x y}-\sigma_{y x}+m_{z}=0,
$$

the deformation measures

$$
\gamma_{x x}=\frac{\partial u}{\partial x}, \gamma_{y x}=\frac{\partial u}{\partial y}+\Omega_{z}, \gamma_{y y}=\frac{\partial v}{\partial y}, \gamma_{x y}=\frac{\partial v}{\partial x}-\Omega_{z}, \kappa_{x z}=\frac{\partial \Omega_{z}}{\partial x},
$$

the constitutive equations

$$
\begin{gathered}
\sigma_{x x}=A_{11} \gamma_{x x}+A_{12} \gamma_{y y}, \sigma_{y y}=A_{12} \gamma_{x x}+A_{22} \gamma_{y y}, \\
\sigma_{y x}=G^{\prime}\left(\gamma_{y x}+\gamma_{x y}\right), \sigma_{x y}=G^{\prime}\left(\gamma_{y x}+\gamma_{x y}\right)+G^{\prime \prime} \gamma_{x y}, \mu_{x z}=B \kappa_{x z},
\end{gathered}
$$

where $E, v$ are the layer Young's modulus, and Poison's ratio, $G^{\prime \prime}=G=E / 2(1+v), b$ is the layer thickness, $\gamma_{j i}, \kappa_{j i}, \sigma_{j i}, \mu_{j i}$ are strains, curvatures, stresses and moment stresses respectively,

$$
A_{11}=A_{22}=\frac{(1-v) E}{(1+v)(1-2 v)}, \quad A_{12}=\frac{v E}{(1+v)(1-2 v)}, \quad B=\frac{E b^{2}}{12\left(1-v^{2}\right)},
$$

and for the case of sliding layers with full contact, $G^{\prime}=0$.

\section{DISLOCATIONS AND DISCLINATIONS IN THE COSSERAT CONTINUUM}

One of the approaches in Fracture Mechanics is to model a crack as a continuous distribution of dislocations, the displacement discontinuities [5]. In the considered medium, except for usual edge dislocations, a disclination, the discontinuity in the Cosserat rotation (Figure 1), should also be taken into consideration. Two edge dislocations with unit Burgers vector (for the $x$-dislocation, the Burgers vector is parallel to $x$-axis and for the $y$-dislocation, the Burgers vector is parallel to $y$ axis) and the disclination (the rotation vector is perpendicular to the $x y$ plane) can exist in the considered 2D medium. In order to pose the corresponding boundary-value problems a cut will be made along the $y$-axis.

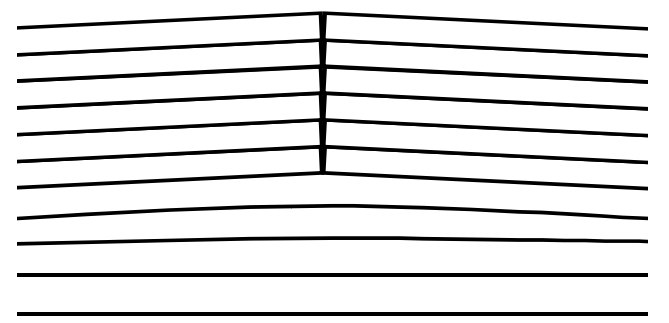

Figure 1: Disclination (discontinuity in the Cosserat rotation).

For the sake of simplicity we will concentrate on the case of sliding layers, $G^{\prime}=0$. In this case the addition of any displacement field $u(x, y)=u^{0}(y), v=0$ will not change the stress state. Therefore, the $x$-dislocation will yield zero stresses. After using the symmetry considerations we obtain the 
following boundary conditions:

$$
\begin{aligned}
& v(0, y)=\left\{\begin{array}{l}
1 / 2, y>0 \\
0, y<0
\end{array}, \sigma_{x x}(0, y)=0, \mu_{x z}(0, y)=0 \text { for the } y\right. \text {-dislocation, } \\
& \Omega_{z}(0, y)=\left\{\begin{array}{l}
1 / 2, y>0 \\
0, y<0
\end{array}, u(0, y)=0, \sigma_{x y}(0, y)=0\right. \text { for the disclination. }
\end{aligned}
$$

Since the introduced Cosserat continuum is only a homogenised model of the layered material, distances smaller than the volume element size (which is supposed to be much larger than the layer thickness) cannot be distinguished. Hence, only the asymptotic solutions corresponding to the distances from the dislocation kernels larger than the layer thickness can be relevant to the original discontinuous material. For further analysis we will be particularly interested in the stress and moment stress behaviour along the $y$-axis $(x=0)$. It can be shown that the main term for the corresponding asymptotics of large $y(y / b \rightarrow \infty), x=0$ for the unity dislocation is obtained as follows

$$
\sigma_{x y}^{\text {disloc }}(0, y)=-\frac{E b^{1 / 2}}{8\left(1-v^{2}\right) \sqrt{2 \pi} 3^{1 / 4}} \frac{\operatorname{sgn} y}{\sqrt{|y|^{3}}}+O\left(\frac{b^{5 / 2}}{|y|^{5 / 2}}\right), \sigma_{y y}^{\text {disloc }}(0, y)=\frac{E \delta(y)}{2\left(1-v^{2}\right)}
$$

all other fields are zero. The main asymptotic terms of large $y, x=0$ for the unit disclination are:

$$
\begin{aligned}
& \sigma_{y y}^{\text {disclin }}(0, y)=-\frac{E b^{1 / 2}}{4\left(1-v^{2}\right) \sqrt{2 \pi} 3^{1 / 4}} \frac{1}{\sqrt{|y|}}+O\left(\frac{b^{3 / 2}}{|y|^{3 / 2}}\right), \\
& \mu_{x z}^{\text {disclin }}(0, y)=-\frac{E b^{3 / 2} 3^{1 / 4}}{24\left(1-v^{2}\right) \sqrt{2 \pi}} \frac{\operatorname{sgn} y}{\sqrt{|y|}}+O\left(\frac{b^{3 / 2}}{|y|^{3 / 2}}\right),
\end{aligned}
$$

all other stress components being zero on the $y$-axis.

\section{BENDING CRACK - A NEW FRACTURE MODE}

The Cosserat continuum is characterised by the presence of three additional degrees of freedom corresponding to three components of independent Cosserat rotation. Consequently, three more crack modes (Modes IV - VI) can exist. These cracks can be envisaged as discontinuities in the corresponding components of the Cosserat rotation (Figure 1) and modelled as distributions of disclinations (or disclination loops for 3D cracks).

As we found above, the disclination does not create shear stresses along the axis perpendicular to the direction of layering, while the dislocation does not create a moment stress along the same axis. This allows us to consider a crack of the new mode along the $y$-axis which does not mix with and is independent of the classical crack modes. This crack is located along the $y$-axis and is composed of some continuous distribution of the disclinations, the same way as cracks of the classical modes are represented by corresponding continuous dislocation distributions. Since the disclination is a jump (discontinuity) in the Cosserat rotation which in its turn presents the continuum description of the layers deflection, we will call such a crack the bending crack. Since the layer bending is represented by the Cosserat rotation component parallel to the $z$-axis, the bending crack corresponds to Mode VI of the above classification.

For the layered material with a semi-infinite pure bending crack at $x=0, y>0$ which faces are loaded by moment stress, $\mu_{x z}{ }^{0}(y)$, the following integral equation can be written 


$$
C \int_{0}^{\infty} \frac{\operatorname{sgn}(y-t) \Delta \Omega_{z}^{\prime}(t)}{\sqrt{|y-t|}} d t=\mu_{x z}{ }^{0}(y), \quad y \geq 0, \quad C=-\frac{E b^{3 / 2} 3^{1 / 4}}{24\left(1-v^{2}\right) \sqrt{2 \pi}} .
$$

For the moment stresses on the crack continuation $(x=0, y<0)$ the physically admissible (allowing finite energy) eigenmode which produces a singularity is

$$
\Omega_{z}^{\prime}(y) \sim y^{-3 / 4}, \quad \Omega_{z}(y) \sim y^{1 / 4}, \quad \mu_{x z} \sim(-y)^{-1 / 4} .
$$

Thus a new fracture mode emerges in layered materials. It corresponds to the discontinuity of rotations (derivatives of the layer deflection) and produces a concentration of moment stresses (previously, only conventional cracks which are displacement discontinuities were considered in the Cosserat continua, eg, [6, 7]). It is characterized by the moment stress intensity factor at the crack tip

$$
\mathrm{M}_{z} \stackrel{\operatorname{def}}{=} \lim _{y \rightarrow-0} \mu_{x z}(-y) \sqrt[4]{-y}, \quad \mu_{x z} \sim \frac{\mathrm{M}_{z}}{(-y)^{1 / 4}}, \quad y \rightarrow-0 .
$$

A criterion of growth of pure bending crack can be formulated in terms of the critical value of the moment stress intensity factor (the coefficient at the moment stress singularity) or using the usual energy criterion. However, the presence of the minimal length (the layer thickness, $b$ ) and the fact that the moment stress concentration is not strong and slowly decaying suggest a criterion based on the critical moment stress at a distance $b$ from the crack tip. In the case when the action of conventional stresses can be neglected and the layers are brittle, the critical value of the moment stress is determined by the value of the bending-generated normal stress created at a layer edge necessary to start its fracture. If $\sigma_{t}$ is the local tensile strength, the criterion will read

$$
\mu_{x z}(b)=\mu_{c r}=\frac{b \sigma_{t}}{6\left(1-v^{2}\right)}
$$

or, in terms of the moment stress intensity factor (12)

$$
\mathrm{M}_{z}=\mathrm{M}_{z c} \stackrel{\operatorname{def}}{=} \frac{b^{5 / 4} \sigma_{\tau}}{6\left(1-v^{2}\right)} .
$$

The criterion of bending crack growth can also be formulated in terms of the energy balance. If, for instance, a Barenblatt-type process zone is introduced as a zone of a specified dependence of the moment stress $\mu(\delta)$ versus rotation discontinuity, the energy flux necessary to start crack growth (for a crack along y-axis) is given by (see $[4,8]$ for details):

$$
\Pi_{y}=-\int_{0}^{\delta_{*}} \mu(\delta) d \delta,
$$

where $\delta_{*}$ is the maximum rotation discontinuity. This is an analogue of the path-independent $\mathrm{J}$ integral for the Cosserat continuum under consideration.

\section{SEMI-INFINITE BENDING CRACK}

Equation (10) was solved in [4] using the Wiener-Hopf method. For a point moment $\mu_{x z}{ }^{0}(y)=\delta(y$ $a), a>0$ the solution will play the role of the Green's function. The solution of (10) reads:

$$
2 \pi C_{1} \Delta \Omega_{z}^{\prime}(t)=\frac{1}{2} \frac{\operatorname{sgn}(t-a)}{|t-a|^{3 / 2}}+\frac{1}{2 \pi} \frac{1}{\sqrt{2} a^{3 / 2}} K r\left(\frac{t}{a}\right)+\widetilde{C} \frac{1}{t^{3 / 4}}, t>0, C_{1}=-C,
$$




$$
\operatorname{Kr}(x)=\int_{0}^{+\infty} \frac{d \tau}{\tau^{1 / 4}(1+\tau)(1+\tau x)^{3 / 2}} .
$$

Function $\operatorname{Kr}(x)$ has the following asymptotic behaviour

$$
\operatorname{Kr}(x)=\frac{I_{0}}{x^{3 / 4}}+O\left(\frac{1}{x^{7 / 4}}\right), x \rightarrow+\infty, I_{0}=\int_{0}^{+\infty} \frac{d y}{y^{1 / 4}(1+y)^{3 / 2}}=\frac{4 \pi^{3 / 2}}{\Gamma(1 / 4)^{2}} \approx 1.695 .
$$

The asymptotics of the solution as $t \rightarrow+\infty$

$$
2 \pi C_{1} \Delta \Omega_{z}^{\prime}(t) \underset{t \rightarrow+\infty}{\sim} \frac{1}{2 \pi} \frac{1}{\sqrt{2} a^{3 / 4}} \frac{I_{0}}{t^{3 / 4}}+\frac{\widetilde{C}}{t^{3 / 4}}+O\left(\frac{1}{t^{3 / 2}}\right)
$$

shows that in order to avoid the infinite value of the discontinuity in the Cosserat rotation $\Delta \Omega$ at infinity, one has to choose $\widetilde{C}=-I_{0} 2^{-3 / 2} a^{-3 / 4} / \pi$. Since in (16) the crack tip singularity is only given by the term with $\widetilde{C}$, the moment stress intensity factor is

$$
\mathrm{M}_{z}=\frac{\widetilde{C}}{\sqrt{2 \pi}} \frac{\Gamma(1 / 4)}{\Gamma(3 / 4)}=-\frac{\sqrt{2}}{2 \pi a^{3 / 4}} \approx-\frac{0.225}{a^{3 / 4}} .
$$

The energy release rate can be expressed through $M_{z}$ (see [4] for details):

$$
\mathrm{E}=\frac{3^{3 / 4} \pi^{2}\left(1-v^{2}\right)}{4 E b^{3 / 2}(\Gamma(1 / 4))^{2}} M_{z}^{2} .
$$

The comparison of the energy release rate with (15) gives the energy criterion of bending crack growth.

\section{FINITE BENDING CRACK}

Next we consider a finite bending crack perpendicular to the direction of layering at $x=0,-1<y<1$. For the faces loaded with the arbitrary by moment stress $\mu_{x z}{ }^{0}(y)$ the following integral equation can be written

$$
C \int_{-1}^{1} \frac{\operatorname{sgn}(y-t) \Delta \Omega_{z}^{\prime}(t)}{\sqrt{|y-t|}} d t=\mu_{x z}{ }^{0}(y), \quad-1<y<1, \quad C=-\frac{E b^{3 / 2} 3^{1 / 4}}{24\left(1-v^{2}\right) \sqrt{2 \pi}} .
$$

The constant $\mathrm{C}$ is the same as in (10). The integral equation (21) is the Abel's type integral equation. Its solution reads (eg, [9]):

$$
C \Delta \Omega_{z}^{\prime}(t)=\frac{1}{2 \pi} \frac{d}{d t} \int_{-1}^{t} \frac{\mu_{x z}^{0}(\xi)}{\sqrt{t-\xi}} d \xi+\frac{1}{2 \pi^{2}\left(1-t^{2}\right)^{3 / 4}} \int_{-1}^{1} \frac{\left(1-\xi^{2}\right)^{3 / 4}}{\xi-t} d \xi \frac{d}{d \xi} \int_{-1}^{\xi} \frac{\mu_{x z}^{0}(\omega)}{(\xi-\omega)^{1 / 2}} d \omega+\frac{\widetilde{C} \sqrt{2}}{2 \pi\left(1-t^{2}\right)^{3 / 4}},
$$

Let us suppose that the crack faces are subjected to the uniformly distributed moment stress $M$ along the crack, ie $\mu_{x z}{ }^{0}(y)=M,-1<y<1$. Then the solution (22) reads

$$
C \Delta \Omega_{z}^{\prime}(t)=M \frac{\sqrt{2}}{2 \pi} \cdot \frac{-t+1 / 2}{\left(1-t^{2}\right)^{3 / 4}}+\frac{\widetilde{C} \sqrt{2}}{2 \pi\left(1-t^{2}\right)^{3 / 4}},-1<t<1 .
$$

Constant $\widetilde{C}$ can be found from the singlevaluedness of the Cosserat rotation at passing around the crack

$$
\int_{-1}^{1} \Delta \Omega_{z}^{\prime}(t) d t=0 \Rightarrow \widetilde{C}=-M / 2
$$


Therefore, the moment stress intensity factor is $\mathrm{M}_{z}= \pm M(\Gamma(1 / 4))^{2} \pi^{-3 / 2} 2^{-1 / 4}$, for $t= \pm 1$. For a crack of length $2 a$ the dimension analysis gives $\mathrm{M}_{2}= \pm M(\Gamma(1 / 4))^{2} \pi^{-3 / 2} 2^{-1 / 4} a^{1 / 4}$, for $t= \pm a$.

\section{CONCLUSION}

In Cosserat continuum modelling layered materials with freely sliding layers, the dislocations and disclinations create stresses that concentrate on an axis normal to the layering. As a result the bending fracture zone will propagate normally to the layering. The moment stresses may create a fracture that develops as a progressive rupture of layers in a narrow zone propagating as a "bending crack". This crack being a distribution of discontinuities in Cosserat rotation presents a new fracture mode that is independent and can be separated from the classical fracture Modes I-III.

The concentration of bending moments at the tip of the bending crack is singular increasing as $r^{-1 / 4}$, where $r$ is the distance from the crack tip. This is weaker than the stress singularity at the tip of a conventional crack. This singularity is governed by the moment stress intensity factor that in the case of finite crack uniformly loaded by distributed moments is proportional to $1 / 4$ power of the crack length. Both energy release rate and the criterion of crack growth can be formulated through the moment stress intensity factor.

Acknowledgment. The first author acknowledges the support of the Australian Research Council Australian Postdoctoral Fellowship and Discovery Grant, DP0346148 (2003-2006).

\section{REFERENCES}

1. Zvolinskii, N.V. and Shkhinek, K.N. Continual model of laminar elastic medium, Mechanics of Solids, 19(1), 1-9 (1984).

2. Mühlhaus, H.-B. Continuum models for layered and blocky rock. In: Comprehensive Rock Eng., Invited Chapter for Vol. II: Analysis and Design Methods, Pergamon Press, 209-230 (1993).

3. Mühlhaus, H.-B. A relative gradient model for laminated materials. In: Continuum Models for Materials with Microstructure, Mühlhaus, H.-B., (Ed.), Chapter 13, John Wiley \& Sons, 451482 (1995).

4. Pasternak, E. Generalised homogenisation procedures for granular and layered materials, $\mathrm{PhD}$ thesis, The University of Western Australia (2002).

5. Bilby, B.A. and Eshelby, J.D. Dislocation and the Theory of Fracture. In: Fracture, An Advanced Treatise, Liebowitz, H., (Ed). I, Microscopic and Macroscopic Fundamentals, Academic Press, New York and London, 99-182 (1968).

6. Sternberg, E. and Muki, R. The effect of couple stress on the stress concentration of a crack, Int. J. Solids Struct., 3, 69-95 (1967).

7. Atkinson, C. and Leppington, F.G. The effect of couple stresses on the tip of a crack, Int. J. Solids Structures, 13, 1103-1122 (1977).

8. Mühlhaus, H.-B. and Pasternak, E. Path-independent integrals for Cosserat Continuum, International Journal of Fracture 113 (4): L21-L26 (2002).

9. Estrada, R. and Kanwal, R.P. (2000) Singular Integral Equations, Birkhäuser, Boston. 